\title{
Planned Time Point Number
}

National Cancer Institute

\section{Source}

National Cancer Institute. Planned Time Point Number. NCI Thesaurus. Code C82545.

Numeric description of a specific moment in time. 